
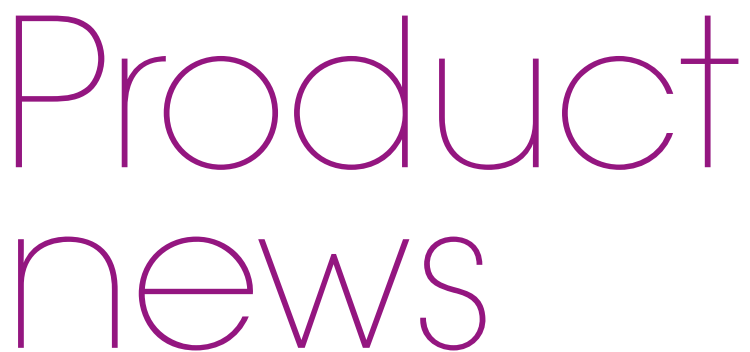

\title{
PROMOTING BENEFICIAL BACTERIA AND REDUCING DISEASE
}

When it comes to oral health, dental probiotics have shown potential to rebalance the oral microbiome by crowding out the 'bad bacteria' and promoting the beneficial bacteria, in turn reducing the risk of dental disease. Dental probiotics are emerging as a promising concept in treatment of gum disease; they also help reduce the risk of dental decay by reducing levels of certain decay-causing bacteria. They display anti-inflammatory properties, helping to improve gum health and reduce bleeding from the gums. There is also evidence that dental probiotics manage smelly breath, by suppressing the production of bacteria producing volatile sulphur compounds that contribute to oral malodour.

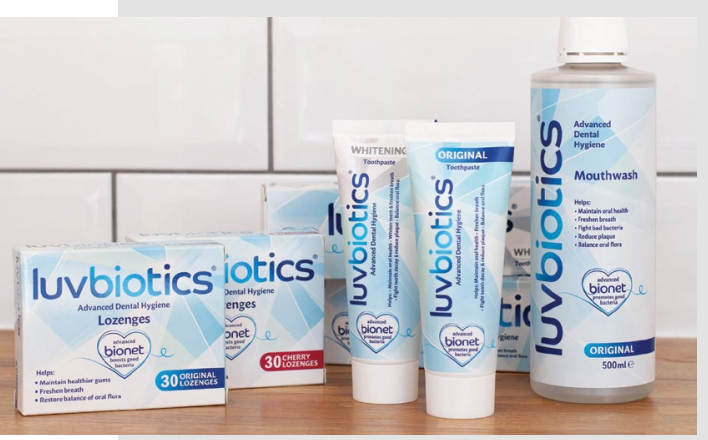

The antimicrobial and anti-inflammatory effects of dental probiotics not only benefit the oral microbiome but also have further positive impact on the rest of the body. Just as good gut health affects the entire body, a balanced oral microbiome supports gut health and general immunity.

The Luvbiotics range, recently launched in the UK, uses bionet technology powered by probiotics and xylitol with natural ingredients to promote good bacteria and balance oral flora for a happier, healthier mouth and improved wellbeing.

The Luvbiotics range has been developed using pioneering breakthrough technology for over two years with a world leading research institute, scientists and dentists.

The range of products includes toothpaste, mouthwash and lozenges: Luvbiotics Original Toothpaste, Luvbiotics Whitening Toothpaste, Luvbiotics Lozenges Cherry, Luvbiotics Lozenges Original and Luvbiotics Mouthwash Original. They are all available through Amazon. The Luvbiotics toothpastes contain $0.32 \% \mathrm{w} / \mathrm{w}$ sodium fluoride and the mouthwash contains $0.05 \%$ $\mathrm{w} / \mathrm{w}$ sodium fluoride in the formulation.

For more information visit https://www. luvbiotics.co.uk/.
If you would like to promote your products or services direct to the dental industry in BDJ Team, call Andy May on 02078434785 or emaila.may@nature.com.

\section{FLAGSHIP EVENT SET FOR GLASGOW}

The British Society of Dental Hygiene and Therapy (BSDHT) is excited to announce that its flagship event, the Oral Health Conference (OHC) will be taking place in Glasgow on 26-27 November 2021.

The $\mathrm{OHC}$ is the perfect opportunity for dental hygienists, dental therapists and students of the profession to network with likeminded individuals, keep up to date with the latest research in the field and expand in their careers.

As always, the show will see a number of exciting lectures, hands-on workshops and product demonstrations, plus offer the chance to catch up with colleagues and meet new people who share your passions and interests.

So, make sure to put the date in your diaries if you don't want to miss out!

For more information about the BSDHT, visit www.bsdht.org.uk, call 01788 575050 or email enquiries@bsdht.org.uk.

\section{HIGHLY EFFICIENT INSTRUMENT PROCESSING}

Manufacturer W\&H is proud to introduce the new Teon thermal washer disinfector, offering exceptional performance for highly efficient cleaning and reprocessing of dental instruments. With its highcapacity chamber, the Teon is optimised to accommodate a larger throughput of instruments, and can easily integrate into your decontamination workflow, given its user-friendly interface and dedicated custom programmes. To promote maximum user confidence, the Teon also comes with a range of additional safety features. This includes an integrated spray arm monitoring system, a speed variable pump, an advanced filter system, and an automatic door lock.

Discover how to enhance the cost-efficiency of dental decontamination with Teon. To find out more visit www.wh.com/en_uk, call 01727 874990 or email office.uk@wh.com.

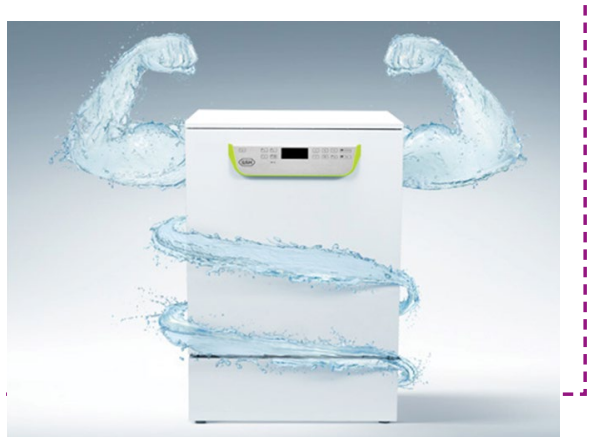

